\title{
A REFINED WARING PROBLEM FOR FINITE SIMPLE GROUPS
}

\author{
MICHAEL LARSEN ${ }^{1}$ and PHAM HUU TIEP ${ }^{2}$ \\ ${ }^{1}$ Department of Mathematics, Indiana University, Bloomington, IN 47405, USA; \\ email: mjlarsen@indiana.edu \\ ${ }^{2}$ Department of Mathematics, University of Arizona, Tucson, AZ 85721, USA; \\ email: tiep@math.arizona.edu
}

Received 28 July 2014; accepted 23 February 2015

\begin{abstract}
Let $w_{1}$ and $w_{2}$ be nontrivial words in free groups $F_{n_{1}}$ and $F_{n_{2}}$, respectively. We prove that, for all sufficiently large finite nonabelian simple groups $G$, there exist subsets $C_{1} \subseteq w_{1}(G)$ and $C_{2} \subseteq$ $w_{2}(G)$ such that $\left|C_{i}\right|=O\left(|G|^{1 / 2} \log ^{1 / 2}|G|\right)$ and $C_{1} C_{2}=G$. In particular, if $w$ is any nontrivial word and $G$ is a sufficiently large finite nonabelian simple group, then $w(G)$ contains a thin base of order 2. This is a nonabelian analog of a result of Van $\mathrm{Vu}$ ['On a refinement of Waring's problem', Duke Math. J. 105(1) (2000), 107-134.] for the classical Waring problem. Further results concerning thin bases of $G$ of order 2 are established for any finite group and for any compact Lie group $G$.
\end{abstract}

2010 Mathematics Subject Classification: 20C33 (primary); 20D06 (secondary)

\section{Introduction}

Let $F_{n}$ denote the free group in $n$ generators and $w \in F_{n}$ a nontrivial element. For every group $G$, the word $w$ induces a function $G^{n} \rightarrow G$, which we also denote $w$. In joint work with Aner Shalev [LS2, LST], the authors proved that, if $G$ is a finite simple group whose order is sufficiently large in terms of $w$, then $w\left(G^{n}\right)$ is a basis of order 2; that is, every element of $G$ can be written as the product of two elements of $w\left(G^{n}\right)$. In particular, for any positive integer $m$, the $m$ th powers in $G$ form a basis of order 2 for all sufficiently large finite simple groups; this example explains the use of the term 'Waring problem' in the title of this paper. 
The refinement we have in mind is indicated by a result of $\mathrm{Van} \mathrm{Vu}$ [ $\mathrm{Vu}$ ] on the classical Waring problem. Vu observed that the $m$ th powers in the set $\mathbb{N}$ of natural numbers form a thick basis of sufficiently large order $s$, in the sense that the number of representations of $n \in \mathbb{N}$ as a sum of $s m$ th powers grows polynomially with $n$. He proved that the $m$ th powers contain thin subbases of order $s$, that is, subsets $X$ for which every element of $\mathbb{N}$ can be written as a sum of $s$ elements of $X$, but the growth of the number of representations is logarithmic. He asked one of us if there is an analogous result in the group-theoretic setting, that is, if $w\left(G^{n}\right)$ contains a thin subbase of order 2 . The main result of this paper gives an affirmative answer to this question; in fact, the growth of the average number of representations of $g \in G$ is $O(\log |G|)$.

More precisely, our result is as follows. We state it asymmetrically, that is, in the more general case that we have two possibly different words $w_{1}$ and $w_{2}$ instead of a single word $w$.

THEOREM 1.1. Let $w_{1}$ and $w_{2}$ be nontrivial words in free groups $F_{n_{1}}$ and $F_{n_{2}}$, respectively. For all sufficiently large finite nonabelian simple groups $G$, there exist subsets $C_{1} \subseteq w_{1}(G)$ and $C_{2} \subseteq w_{2}(G)$ such that $\left|C_{i}\right|=O\left(|G|^{1 / 2} \log ^{1 / 2}|G|\right)$ and $C_{1} C_{2}=G$.

It is known that, for many words $w$, we have $w\left(G^{n}\right)=G$ for all $G$ sufficiently large. For instance, the commutator word in $F_{2}$ satisfies this equality for all finite simple $G$; see [EG], [LBST]. In this case, we are looking for a thin subbase of $G$ itself, and we prove that such order-2 subbases $X_{G}$ exist, not merely for finite simple groups but for all finite groups, where the average number of representations of $G$ as a product of two elements in $X_{G}$ is $O(1)$ as $|G| \rightarrow \infty$; see Corollary 5.4. We conclude with an analogous result for compact Lie groups; see Proposition 6.4 and Theorem 6.5.

\section{The probabilistic method}

Given subsets $X$ and $Y$ of a finite group $G$ with $X Y=G$, we would like to find subsets $X_{0} \subseteq X$ and $Y_{0} \subseteq Y$ such that $X_{0} Y_{0}$ is still all of $G$, while $\left|X_{0}\right|\left|Y_{0}\right|$ is only slightly larger than $|G|$. In this section, we show that appropriately large random subsets $X_{0} \subseteq X$ and $Y_{0} \subseteq Y$ usually have the property that $X_{0} Y_{0}$ includes every element of $G$ that has many representations of the form $x y, x \in X, y \in Y$.

LeMma 2.1. Let $a, b, n$ be positive integers, $N$ a set of cardinality $n, A \subseteq N a$ fixed subset of cardinality $a$, and $B \subseteq N$ a random subset chosen uniformly from 
all b-element subsets of $N$. Then

$$
\operatorname{Pr}[A \cap B=\emptyset] \leqslant e^{-a b / n} .
$$

Proof. The statement is trivial if $a+b>n$, so we assume that $a+b \leqslant n$. The probability that $A \cap B=\emptyset$ is

$$
\begin{aligned}
\frac{\left(\begin{array}{c}
n-a \\
b
\end{array}\right)}{\left(\begin{array}{c}
n \\
b
\end{array}\right)} & =\frac{(n-a) !(n-b) !}{n !(n-a-b) !}=\frac{(n-a)(n-a-1) \cdots(n-a-b+1)}{n(n-1) \cdots(n-b+1)} \\
& \leqslant(1-a / n)^{b} \leqslant e^{-a b / n} .
\end{aligned}
$$

The following lemma gives a somewhat cruder but more general estimate than Lemma 2.1.

LEMMA 2.2. Let $a, b, n$ be positive integers, $N$ a set of cardinality $n, A \subseteq N a$ fixed subset of cardinality $a$, and $B \subseteq N$ a random subset chosen uniformly from all b-element subsets of $N$. Then

$$
\operatorname{Pr}\left(|A \cap B| \leqslant \frac{a b}{e^{2} n}\right) \leqslant(2.2) e^{-5 a b / 2 e^{2} n} .
$$

Proof. Assume that $\max (a+b-n, 0) \leqslant k \leqslant \min (a, b)$ so that $k$ is a possible size for $A \cap B$. For $k>0$ we have $k !>(k / e)^{k}$, and so the probability that $|A \cap B|=k$ is

$$
\begin{aligned}
\frac{\left(\begin{array}{l}
a \\
k
\end{array}\right)\left(\begin{array}{l}
n-a \\
b-k
\end{array}\right)}{\left(\begin{array}{l}
n \\
b
\end{array}\right)} & =\frac{a ! b !(n-a) !(n-b) !}{k !(a-k) !(b-k) ! n !(n-a-b+k) !} \\
& =\frac{b \cdots(b-k+1)}{k \cdots(a-k+1)} \frac{(n-a) \cdots(n-a-b+k+1)}{n \cdots(n-k+1)} \frac{(n-k) \cdots(n-b+1)}{(b-k)(a-k)} \\
& <\frac{b^{k}}{(k / e)^{k}} \frac{a^{k}}{n^{k}} \frac{(n-a)^{b-k}}{(n-k)^{b-k}} \leqslant \frac{(a b / n)^{k}}{(k / e)^{k}} \exp \left(-\frac{(b-k}{}\right) \\
& =\exp (f(k)),
\end{aligned}
$$

where

$$
f(x):=x+x \log a b / n-x \log x-g(x), \quad g(x):=(a-x)(b-x) /(n-x) .
$$

Let $r:=a b / e^{2} n \leqslant \min \left(a / e^{2}, b / e^{2}\right)$. Then, when $0<x \leqslant r$, we have $f^{\prime}(x)>2$, and so $f(x)$ is increasing on $(0, r]$, and $f(x)-f(x-1)>2$ when $1<x \leqslant r$. Also,

$$
g(r) \geqslant \frac{a b\left(1-e^{-2}\right)^{2}}{n}>5.5 r, \quad f(r)=3 r-g(r)<-2.5 r
$$


It follows that

$$
\begin{aligned}
\operatorname{Pr}(0<|A \cap B| \leqslant r) & \leqslant \sum_{i=1}^{\lfloor r\rfloor} \exp (f(i))<\frac{1}{1-e^{-2}} \exp (f(r)) \\
& <\frac{e^{-2.5 r}}{1-e^{-2}}<(1.2) e^{-2.5 r} .
\end{aligned}
$$

Together with Lemma 2.1, this implies the claim.

Proposition 2.3. Let $c>0$ be a constant, and let $X, Y$, and $Z$ be subsets of a finite group $G$ such that, for all $z \in Z$,

$$
|\{(x, y) \in X \times Y \mid x y=z\}| \geqslant \frac{c|X||Y|}{|G|} .
$$

Let $x_{0} \leqslant|X|$ and $y_{0} \leqslant|Y|$ be positive integers such that

$$
x_{0} y_{0} \geqslant\left(2 e^{2} / c\right)|G| \log |G| \text {. }
$$

Then there exist subsets $X_{0} \subseteq X$ and $Y_{0} \subseteq Y$, with $x_{0}$ and $y_{0}$ elements, respectively, such that $X_{0} Y_{0} \supseteq Z$.

Proof. Let $n$ denote the order of $G$, which we may assume is at least 2 . We choose $X_{0}$ and $Y_{0}$ at random independently and uniformly from the subsets of $X$ of cardinality $x_{0}$ and the subsets of $Y$ of cardinality $y_{0}$, respectively. It suffices to prove that, for each $z \in Z$, the probability that $z \in X_{0} Y_{0}$ is more than $1-1 / n$. (Indeed, in this case the probability that $X_{0} Y_{0}=G$ is larger than $1-n / n=0$; that is, $X_{0} Y_{0}=G$.) Let $S_{z}$ denote the set of pairs $(x, y) \in X \times Y$ such that $x y=z$, and let $\pi_{X}$ and $\pi_{Y}$ denote the projection maps from $X \times Y$ to $X$ and $Y$, respectively. We want to prove that the probability that $\pi_{Y}^{-1}\left(Y_{0}\right) \cap \pi_{X}^{-1}\left(X_{0}\right) \cap S_{z}$ is nonempty is more than $1-1 / n$.

As $G$ is a group, the restrictions of $\pi_{X}$ and $\pi_{Y}$ to $S_{z}$ are injective, so

$$
\begin{gathered}
\left|\pi_{X}^{-1}\left(X_{0}\right) \cap S_{z}\right|=\left|\pi_{X}\left(S_{z}\right) \cap X_{0}\right|, \\
\left|\pi_{Y}^{-1}\left(Y_{0}\right) \cap \pi_{X}^{-1}\left(X_{0}\right) \cap S_{z}\right|=\left|\pi_{Y}\left(\pi_{X}^{-1}\left(X_{0}\right) \cap S_{z}\right) \cap Y_{0}\right| .
\end{gathered}
$$

It suffices to prove that the probability that $\pi_{X}\left(S_{z}\right) \cap X_{0}$ has at least $\left(x_{0}\left|S_{z}\right|\right) /\left(e^{2}|X|\right)$ elements is at least $1-1 / 2 n$, and that the conditional probability that $\pi_{Y}\left(\pi_{X}^{-1}\left(X_{0}\right) \cap S_{z}\right) \cap Y_{0}$ is nonempty given that

$$
\left|\pi_{X}\left(S_{z}\right) \cap X_{0}\right| \geqslant \frac{x_{0}\left|S_{z}\right|}{e^{2}|X|}
$$

is at least $1-1 / 2 n$. 
By hypothesis,

$$
\frac{\left|X_{0}\right|\left|\pi_{X}\left(S_{z}\right)\right|}{|X|}=\frac{x_{0}\left|S_{z}\right|}{|X|} \geqslant \frac{c x_{0}|Y|}{n} \geqslant \frac{c x_{0} y_{0}}{n} \geqslant 2 e^{2} \log n .
$$

By Lemma 2.2, the probability that

$$
\left|X_{0} \cap \pi_{X}\left(S_{z}\right)\right|=\left|\pi_{X}^{-1}\left(X_{0}\right) \cap S_{z}\right| \leqslant \frac{x_{0}\left|S_{z}\right|}{e^{2}|X|}
$$

is at most $2.2 / n^{5}<1 / 2 n$. If (2.1) holds, then

$$
\frac{\left|Y_{0}\right|\left|\pi_{X}^{-1}\left(X_{0}\right) \cap S_{z}\right|}{|Y|} \geqslant \frac{x_{0} y_{0}\left|S_{z}\right|}{e^{2}|X||Y|} \geqslant \frac{2 n \log n\left|S_{z}\right|}{c|X||Y|} \geqslant 2 \log n .
$$

By Lemma 2.1, the probability of $Y_{0}$ being disjoint from a subset of $Y$ of cardinality at least $\left(x_{0}\left|S_{z}\right|\right) /\left(e^{2}|X|\right)$ is at most $1 / n^{2} \leqslant 1 / 2 n$.

COROLlARY 2.4. Let $w_{1}$ and $w_{2}$ be two nontrivial words, and let $S$ be a finite simple group. To prove Theorem 1.1 for $\left(w_{1}, w_{2}, S\right)$, it suffices to show that there exist subsets $X \subseteq w_{1}(S), Y \subseteq w_{2}(S)$, and a subset $S_{1} \subset S$ of cardinality at most $|S|^{1 / 2}$, such that the following hold.

(i) $w_{1}(S) w_{2}(S)=S$.

(ii) $|\{(x, y) \in X \times Y \mid x y=g\}| \geqslant \frac{|X| \cdot|Y|}{2|S|}$ for all $g \in S \backslash S_{1}$.

(iii) $|X|,|Y| \geqslant 2 e|S|^{1 / 2} \log ^{1 / 2}|S|$.

Proof. Choose $x_{0}=y_{0}:=\left\lfloor 2 e|S|^{1 / 2} \log ^{1 / 2}|S|\right\rfloor$ (note that we still have $x_{0} \leqslant|X|$ and $\left.y_{0} \leqslant|Y|\right)$. By Proposition 2.3 with $c=1 / 2$, there exist subsets $X_{0} \subseteq X$ and $Y_{0} \subseteq Y$ with $X_{0} Y_{0} \supseteq S \backslash S_{1},\left|X_{0}\right|=x_{0}$, and $\left|Y_{0}\right|=y_{0}$. For each $z \in S_{1}$, by (i) there exists $\left(x_{z}, y_{z}\right) \in w_{1}(S) \times w_{2}(S)$ such that $z=x_{z} y_{z}$. Now set

$$
C_{1}:=X_{0} \cup\left\{x_{z} \mid z \in S_{1}\right\}, \quad C_{2}:=Y_{0} \cup\left\{y_{z} \mid z \in S_{1}\right\}
$$

COROLlaRY 2.5. If $x_{0}$ and $y_{0}$ are integers in $[1,|G|]$ such that $x_{0} y_{0}>$ $2 e^{2}|G| \log |G|$, then there exist subsets $X_{0}$ and $Y_{0}$ of $G$ of cardinality $x_{0}$ and $y_{0}$, respectively, such that $X_{0} Y_{0}=G$.

Proof. Set $X=Y=Z:=G$ and $c=1$ in Proposition 2.3. 
COROLlARY 2.6. There exists a square root $R$ of $G$, that is, a subset such that $R^{2}=G$, with $|R| \leqslant 2^{1 / 2} e|G|^{1 / 2} \log ^{1 / 2}|G|$.

In fact, we will show that $G$ has a square root of size $O\left(|G|^{1 / 2}\right)$; see Corollary 5.4. Analogs of this result for compact Lie groups will be proved in Section 6; cf. Proposition 6.4 and Theorem 6.5.

\section{Simple groups of Lie type}

In what follows, we say that $S$ is a finite simple group of Lie type of rank $r$ defined over $\mathbb{F}_{q}$ if $S=\mathcal{G}^{F} / \mathbf{Z}\left(\mathcal{G}^{F}\right)$ for a simple simply connected algebraic group $\mathcal{G}$ over $\mathbb{F}_{q}$, of rank $r$, and a Steinberg endomorphism $F: \mathcal{G} \rightarrow \mathcal{G}$, with $q$ the common absolute value of the eigenvalues of $F$ on the character group of an $F$-stable maximal torus $\mathcal{T}$ of $\mathcal{G}$. In particular, this includes the Suzuki-Ree groups, for which $q$ is a half-integer power of 2 or 3. By slight abuse of terminology, we will say that an element $s \in S$ is regular semisimple if some inverse image of $s$ is so in $\mathcal{G}^{F}$.

The aim of this section is to prove the following theorem.

THEOREM 3.1. Let $w_{1}$ and $w_{2}$ be two nontrivial words. Then there is $N=N$ $\left(w_{1}, w_{2}\right)$ with the following property. For any finite nonabelian simple group $S$ of Lie type of order at least $N$, there exist conjugacy classes $s_{1}^{S} \subseteq w_{1}(S), s_{2}^{S} \subseteq w_{2}(S)$, and a subset $S_{1} \subset S$ of cardinality at most $|S|^{1 / 2}$, such that the following hold.

(i) $w_{1}(S) w_{2}(S)=S$.

(ii) $\left|\left\{(x, y) \in s_{1}^{S} \times s_{2}^{S} \mid x y=g\right\}\right| \geqslant \frac{\left|s_{1}^{S}\right| \cdot\left|s_{2}^{S}\right|}{2|S|}$ for all $g \in S \backslash S_{1}$.

(iii) $\left|s_{i}^{S}\right| \geqslant 4 e|S|^{1 / 2} \log ^{1 / 2}|S|$.

Note that condition (i) follows from the main result of [LST], and (ii) is equivalent to

$$
\left|\sum_{1_{S} \neq \chi \in \operatorname{Irr}(S)} \frac{\chi\left(s_{1}\right) \chi\left(s_{2}\right) \bar{\chi}(g)}{\chi(1)}\right| \geqslant \frac{1}{2}, \quad \forall g \in S \backslash S_{1} .
$$

Also, Theorem 3.1 and Corollary 2.4 immediately imply Theorem 1.1 for sufficiently large nonabelian simple groups of Lie type.

First we recall the following consequence of [La, Proposition 7]. 
LEMMA 3.2. For any $r_{0}$ and any nontrivial word $w \neq 1$, there exists a constant $c=c\left(w, r_{0}\right)$ such that

$$
|w(S)| \geqslant c|S|
$$

for all finite simple group $S$ of Lie type of rank $\leqslant r_{0}$.

COROLlaRY 3.3. For any $r_{0}$ and any nontrivial word $w \neq 1$, there exists a constant $Q=Q\left(w, r_{0}\right)$ such that

(i) $w(S)$ contains a regular semisimple element s and

(ii) $\left|x^{S}\right| \geqslant 4 e|S|^{1 / 2} \log ^{1 / 2}|S|$ for any regular semisimple element $x \in S$

for all finite simple groups $S$ of Lie type of rank $\leqslant r_{0}$ defined over $\mathbb{F}_{q}$ with $q \geqslant Q$.

Proof. According to [GL, Theorem 1.1], the proportion of regular semisimple elements in $S$ defined over $\mathbb{F}_{q}$ is more than $1-f(q)$, with

$$
f(q):=\frac{3}{q-1}+\frac{2}{(q-1)^{2}} .
$$

Applying Lemma 3.2 and choosing $Q$ so that $f(Q)<c\left(w, r_{0}\right)$, we see that $w(S)$ contains a regular semisimple element $s$ whenever the rank of $S$ is at most $r_{0}$ and $q \geqslant Q$.

Next, view $S$ as $G / \mathbf{Z}(G)$ for $G:=\mathcal{G}^{F}$, and consider an inverse image $g \in G$ of $x$ in $G$ that is regular semisimple. Note that $\left|\mathbf{C}_{G}(g)\right| \leqslant(q+1)^{r}$, and so $\left|\mathbf{C}_{G}(x \mathbf{Z}(G))\right| \leqslant(q+1)^{r}|\mathbf{Z}(G)|$. Also, $|G|>(q-1)^{3 r}$ and $|\mathbf{Z}(G)| \leqslant r_{0}+1$. Therefore,

$$
\left|s^{S}\right|=\frac{|S|}{\left|\mathbf{C}_{S}(x)\right|}=\frac{|G|}{\left|\mathbf{C}_{G}(x \mathbf{Z}(G))\right|} \geqslant \frac{|G|}{(q+1)^{r}\left(r_{0}+1\right)}>|S|^{3 / 5}>4 e|S|^{1 / 2} \log ^{1 / 2}|S|
$$

when $q \geqslant Q$ and we choose $Q$ large enough.

Next we recall the following fact.

LEMMA 3.4. For any $r_{0}$, there is a constant $C=C\left(r_{0}\right)$ such that

$$
|\chi(s)| \leqslant C
$$

for all finite simple group $S$ of Lie type of rank $\leqslant r_{0}$, for all regular semisimple elements $s \in S$, and for all $\chi \in \operatorname{Irr}(S)$. 
Proof. Note that, if $S$ is not a Suzuki-Ree group, then the statement is a direct consequence of [GLL, Proposition 5]. But in fact the same proof goes through in the case that $S$ is a Suzuki-Ree group.

Proposition 3.5. Theorem 3.1 holds for Suzuki and Ree groups, with $S_{1}=\{1\}$.

Proof. Let $S={ }^{2} B_{2}\left(q^{2}\right),{ }^{2} G_{2}\left(q^{2}\right)$, or ${ }^{2} F_{4}\left(q^{2}\right)$. By [LST, Proposition 6.4.1] and Corollary 3.3, there exists $Q_{1}=Q\left(w_{1}, w_{2}\right)$ such that $w_{1}(S) w_{2}(S)=S$, and $w_{i}(S)$ contains a regular semisimple element $s_{i}$ satisfying the condition 3.3(ii) for $i=1$, 2 , whenever $q \geqslant Q_{1}$. By Lemma 3.4, there is some $C>0$, independent of $q$, such that $\left|\chi\left(s_{i}\right)\right| \leqslant C$ for all $\chi \in \operatorname{Irr}(S)$ and $i=1,2$. We will now prove that there is some $B>0$, independent of $q$, such that

$$
\sum_{1_{S} \neq \chi \in \operatorname{Irr}(S)} \frac{|\chi(g)|}{\chi(1)} \leqslant \frac{B}{q}
$$

for all $1 \neq g \in S$. Taking $q \geqslant \max \left(Q_{1}, 2 B C^{2}\right)$, we will achieve (3.1).

First let $S={ }^{2} B_{2}\left(q^{2}\right)$ with $q \geqslant \sqrt{8}$. The character table of $S$ is known; see, for example, [Bu]. In particular, $\operatorname{Irr}(S)$ consists of $q^{2}+3$ characters: $1_{S}$, two characters of degree $q\left(q^{2}-1\right) / \sqrt{2}$, and the remaining characters of degree $\geqslant$ $\left(q^{2}-1\right)\left(q^{2}-q \sqrt{2}+1\right)$. Furthermore,

$$
|\chi(g)| \leqslant q \sqrt{2}+1
$$

for all $1_{S} \neq \chi \in \operatorname{Irr}(S)$ and $1 \neq g \in S$. It follows that

$$
\sum_{1_{S} \neq \chi \in \operatorname{Irr}(S)} \frac{|\chi(g)|}{\chi(1)} \leqslant(q \sqrt{2}+1)\left(\frac{2 \sqrt{2}}{q\left(q^{2}-1\right)}+\frac{q^{2}}{\left(q^{2}-1\right)\left(q^{2}-q \sqrt{2}+1\right)}\right)<\frac{5}{q},
$$

as stated.

Next suppose that $S={ }^{2} G_{2}\left(q^{2}\right)$ with $q \geqslant \sqrt{27}$. The character table of $S$ is known; see, for example, [Wa]. In particular, $\operatorname{Irr}(S)$ consists of $q^{2}+8$ characters: $1_{S}$, one character of degree $q^{4}-q^{2}+1$, six characters of degree $\geqslant q\left(q^{2}-1\right)$ $\left(q^{2}-q \sqrt{3}+1\right) / \sqrt{12}$, and the remaining characters of degree $\geqslant q^{6} / 2$. Furthermore, $|\chi(g)| \leqslant \sqrt{\left|\mathbf{C}_{S}(g)\right|} \leqslant q^{3}$ for all $1 \neq g \in S$. It follows that

$$
\begin{aligned}
\sum_{1_{S \neq \chi \in \operatorname{Irr}(S)} \frac{|\chi(g)|}{\chi(1)}} & \leqslant q^{3}\left(\frac{1}{q^{4}-q^{2}+1}+\frac{6 \sqrt{12}}{q\left(q^{2}-1\right)\left(q^{2}-q \sqrt{3}+1\right)}+\frac{q^{2}}{q^{6} / 2}\right) \\
& <\frac{5}{q},
\end{aligned}
$$

as stated. 
Suppose now that $S={ }^{2} F_{4}\left(q^{2}\right)$ with $q \geqslant \sqrt{8}$. The (generic) character table of $S$ is known in principle, but not all character values are given explicitly in [Chevie] (in particular, ten families of characters are not listed therein). On the other hand, according to [FG, Lu2], $\operatorname{Irr}(S)$ consists of $q^{4}+4 q^{2}+17$ characters: $\chi_{0}:=1_{S}$, four characters $\chi_{1,2,3,4}$ of degree

$$
\begin{gathered}
\chi_{1,2}(1)=q\left(q^{4}-1\right)\left(q^{6}+1\right) / \sqrt{2}, \\
\chi_{3}(1)=q^{2}\left(q^{4}-q^{2}+1\right)\left(q^{8}-q^{4}+1\right), \quad \chi_{4}(1)=\left(q^{2}-1\right)\left(q^{4}+1\right)\left(q^{12}+1\right),
\end{gathered}
$$

and the remaining characters of degree $>q^{20} / 48$ (when $q \geqslant \sqrt{8}$ ). The orders $\left|\mathbf{C}_{S}(g)\right|$ are listed in [Chevie]; in particular, $\left|\mathbf{C}_{S}(g)\right|<2 q^{30}$ when $1 \neq g \in S$. It follows that $|\chi(g)|<\sqrt{\left|\mathbf{C}_{S}(g)\right|}<\sqrt{2} q^{15}$, and so

$$
\begin{aligned}
\sum_{\chi_{0,1,2,3} \neq \chi \in \operatorname{Irr}(S)} \frac{|\chi(g)|}{\chi(1)} & <\frac{\sqrt{2} q^{15}\left(q^{4}+4 q^{2}+12\right)}{q^{20} / 48}+\frac{\sqrt{2} q^{15}}{\left(q^{2}-1\right)\left(q^{4}+1\right)\left(q^{12}+1\right)} \\
& <\frac{144}{q}
\end{aligned}
$$

Among all nontrivial conjugacy classes of $S$, there are two classes $g_{1,2}^{S}$ with

$$
\left|\mathbf{C}_{S}\left(g_{1}\right)\right|=q^{24}\left(q^{2}-1\right)\left(q^{4}+1\right), \quad\left|\mathbf{C}_{S}\left(g_{2}\right)\right|=q^{20}\left(q^{4}-1\right),
$$

and all the other ones have centralizers of order $<4 q^{20}$; cf. [Chevie]. Hence if $g \notin\{1\} \cup g_{1}^{S} \cup g_{2}^{S}$ then $\left|\chi_{i}(g)\right|<2 q^{10}$, and so

$$
\sum_{\chi=\chi_{1,2,3}} \frac{|\chi(g)|}{\chi(1)} \leqslant \frac{3 \cdot 2 q^{10}}{q\left(q^{4}-1\right)\left(q^{6}+1\right) / \sqrt{2}}<\frac{10}{q} .
$$

Finally, for $g=g_{1,2}$, using [Chevie] one can check that

$$
\left|\chi_{1,2}(g)\right| \leqslant q\left(q^{6}-q^{4}+1\right) / \sqrt{2}, \quad\left|\chi_{3}(g)\right| \leqslant q^{8}-q^{4}+q^{2},
$$

whence

$$
\sum_{\chi=\chi_{1,2,3}} \frac{|\chi(g)|}{\chi(1)} \leqslant \frac{\sqrt{2} q\left(q^{6}-q^{4}+1\right)}{q\left(q^{4}-1\right)\left(q^{6}+1\right) / \sqrt{2}}+\frac{q^{8}-q^{4}+q^{2}}{\left(q^{2}-1\right)\left(q^{4}+1\right)\left(q^{12}+1\right)}<\frac{1}{q} .
$$

Taken together, (3.3)-(3.5) imply (3.2) for $S={ }^{2} F_{4}\left(q^{2}\right)$.

PROPOSITION 3.6. Theorem 3.1 holds for all (sufficiently large) finite nonabelian simple groups $S$ of Lie type of bounded rank, with $S_{1}=\{1\}$.

Proof. By Proposition 3.5, we may assume that $S$ is not a Suzuki or Ree group. Assume that $S$ is defined over $\mathbb{F}_{q}$ and of rank $\leqslant r_{0}$. Then we view $S$ as $\mathcal{G}^{F} / \mathbf{Z}\left(\mathcal{G}^{F}\right)$ 
for some simple simply connected algebraic group $\mathcal{G}$, of rank $r \leqslant r_{0}$, and some Steinberg endomorphism $F: \mathcal{G} \rightarrow \mathcal{G}$. According to [LS2, Theorem 1.7], $w_{1}(S) w_{2}(S)=S$ when $q$ is large enough. By [LST, Corollary 5.3.3], there exists a positive constant $\delta=\delta\left(w_{1}, w_{2}, r_{0}\right)$ such that, for any $F$-stable maximal torus $\mathcal{T}$ of $\mathcal{G}$, and for $i=1,2$,

$$
\left|\mathcal{T}^{F} \cap w_{i}\left(\mathcal{G}^{F}\right)\right| \geqslant \delta\left|\mathcal{T}^{F}\right| \geqslant \delta(q-1)^{r} .
$$

On the other hand, part (3) of the proof of [Lu1, Theorem 2.1] shows that $\mathcal{T}^{F}$ contains at most $2^{r} r^{2}(q+1)^{r-1}$ nonregular elements. Hence, if we choose

$$
q>\max \left(5,1+3^{r_{0}} r_{0}^{2} / \delta\right)
$$

then $\mathcal{T}^{F} \cap w_{i}\left(\mathcal{G}^{F}\right)$ contains a regular semisimple element. Now we apply this observation to a pair of $F$-stable maximal tori $\mathcal{T}_{1}, \mathcal{T}_{2}$ of $\mathcal{G}$ that is weakly orthogonal in the sense of [LST, Definition 2.2.1], and get regular semisimple elements $s_{i} \in \mathcal{T}^{F} \cap w_{i}\left(\mathcal{G}^{F}\right)$ for $i=1$,2. By [LST, Proposition 2.2.2], if $\chi \in \operatorname{Irr}\left(\mathcal{G}^{F}\right)$ is nonzero at both $s_{1}$ and $s_{2}$, then $\chi$ is unipotent (and so trivial at $\left.\mathbf{Z}\left(\mathcal{G}^{F}\right)\right)$. In this case, the results of [DL] imply that $\chi\left(s_{1}\right)$ does not depend on the particular choice of the element $s_{1}$ of given type, and similarly for $\chi\left(s_{2}\right)$. Also, $\left|s_{i}^{S}\right| \geqslant 4 e|S|^{1 / 2} \log ^{1 / 2}|S|$ if $q>\max \left(Q\left(w_{1}, r_{0}\right), Q\left(w_{2}, r_{0}\right)\right)$; cf. Corollary 3.3.

We claim that we can find such a pair $\mathcal{T}_{1}, \mathcal{T}_{2}$ so that there are $\kappa \leqslant 4$ characters $\chi \in \operatorname{Irr}\left(\mathcal{G}^{F}\right)$ with $\chi\left(s_{1}\right) \chi\left(s_{2}\right) \neq 0$, and moreover $\left|\chi\left(s_{1}\right) \chi\left(s_{2}\right)\right|=1$ for all such $\chi$. Indeed, this can be done with $\kappa=2$ for $\mathcal{G}^{F}$ of type $A_{r}$ by [MSW, Theorem 2.1], of type ${ }^{2} A_{r}$ by [MSW, Theorem 2.2], of type $C_{r}$ by [MSW, Theorem 2.3], of type $B_{r}$ by [MSW, Theorem 2.4], of type ${ }^{2} D_{r}$ by [MSW, Theorem 2.5], and of type $D_{2 l+1}$ by [MSW, Theorem 2.6]. For type $D_{2 l}$, we can get $\kappa=4$ by using [GT, Proposition 2.3]. For the exceptional groups of Lie type, we can get $\kappa=2$ by using [LM, Theorem 10.1]. Certainly, if $\kappa=2$, then these characters are the trivial character and the Steinberg character St of $\mathcal{G}^{F}$.

Now consider any nontrivial element $g \in S$. Since $S$ is simple, St is faithful, and so $|\operatorname{St}(g)|<\operatorname{St}(1)$. But $\operatorname{St}(g) \in \mathbb{Z}$ divides $\operatorname{St}(1)$, so we get $|\operatorname{St}(g) / \operatorname{St}(1)| \leqslant 1 / 2$ and

$$
\sum_{1 S \neq \chi \in \operatorname{Irr}(S)}\left|\frac{\chi\left(s_{1}\right) \chi\left(s_{2}\right) \bar{\chi}(g)}{\chi(1)}\right|=\frac{|\operatorname{St}(g)|}{\operatorname{St}(1)} \leqslant 1 / 2,
$$

as desired. Finally, assume that $\kappa=4$ (so $\mathcal{G}^{F}$ is of type $D_{2 l}$ ). By [LST, Theorem 1.2.1], we have

$$
\sum_{1_{S} \neq \chi \in \operatorname{Irr}(S)}\left|\frac{\chi\left(s_{1}\right) \chi\left(s_{2}\right) \bar{\chi}(g)}{\chi(1)}\right| \leqslant 3 q^{-1 / 481}<1 / 2
$$

if $q>6^{481}$. 
To deal with (classical) groups of unbounded rank, we recall the notion of the support of an element of a classical group [LST, Definition 4.1.1]. For $g \in G L_{n}(\mathbb{F}) \subset G L_{n}(\overline{\mathbb{F}})$, the support is the codimension of the largest eigenspace of $g$ acting on $\mathbb{F}^{n}$. The support of any element in a classical group $G(\mathbb{F})$ is the support of its image under the natural representation $\rho: G(\overline{\mathbb{F}}) \rightarrow G L_{n}(\overline{\mathbb{F}})$. Most elements have large support; we have the following quantitative estimate.

LEMMA 3.7. Let $S$ be a finite simple classical group of rank $r \geqslant 8$, and $B \geqslant 1$ any constant. If $r \geqslant 8 B+3$, then the set $S_{1}$ of elements of support $<B$ can contain at most $|S|^{1 / 2}$ elements of $S$.

Proof. We will bound the total number $N$ of elements $g$ of support $\leqslant B$ in $L=$ $S L_{n}(q), S U_{n}(q), S p_{n}(q)$, or $S O_{n}^{ \pm}(q)$ (note that $S \hookrightarrow L / \mathbf{Z}(L)$ ). Let $V=\mathbb{F}_{q}^{n}$, respectively $\mathbb{F}_{q^{2}}^{n}, \mathbb{F}_{q}^{n}, \mathbb{F}_{q}^{n}$, denote the natural $L$-module. By the results in [FG, Section 3], the number of conjugacy classes in $L$ is less than $16 q^{r} \leqslant q^{r+4}$. Since $B<n / 2, g$ has a primary eigenvalue $\lambda \in \mathbb{F}_{q}^{\times}$, respectively $\lambda^{q+1}=1, \lambda= \pm 1$, or $\lambda= \pm 1$; cf. [LST, Proposition 4.1.2]. Moreover, one can show that $V$ admits a $g$-invariant decomposition $V=U \oplus W$ into a direct (orthogonal if $L \neq S L_{n}(q)$ ) sum of (nondegenerate if $\left.L \neq S L_{n}(q)\right)$ subspaces, with $U \leqslant \operatorname{Ker}\left(g-\lambda \cdot 1_{V}\right)$ and $m:=\operatorname{dim}(U) \geqslant n-2 B$ (see [LST, Lemma 6.3.4] for the orthogonal case).

Consider the case $L=S L_{n}^{\epsilon}(q)$, with $\epsilon=+$ for $S L$ and $\epsilon=-$ for $S U_{n}(q)$. Then $\mathbf{C}_{L}(g)$ contains $S L_{m}^{\epsilon}(q)$. It follows that

$$
\left|g^{L}\right| \leqslant \frac{\left|S L_{n}^{\epsilon}(q)\right|}{\left|S L_{m}^{\epsilon}(q)\right|}<\frac{2 q^{n^{2}-1}}{q^{m^{2}-1} / 2}=4 q^{n^{2}-m^{2}} \leqslant q^{4 n B+2},
$$

as $n \geqslant m \geqslant n-2 B$. Hence,

$$
N \leqslant q^{n(4 B+1)+3} \leqslant q^{\left(n^{2}-3\right) / 2} \leqslant|S|^{1 / 2} .
$$

Suppose now that $L=S O_{n}^{ \pm}(q)$. Then $\mathbf{C}_{L}(g)$ contains $S O_{m}^{ \pm}(q)$. It follows that

$$
\left|g^{L}\right| \leqslant \frac{\left|S O_{n}^{ \pm}(q)\right|}{\left|S O_{m}^{ \pm}(q)\right|}<\frac{q^{n(n-1) / 2}}{q^{m(m-1) / 2} / 2}=2 q^{(n-m)(n+m-1) / 2+1} \leqslant q^{(2 n-1) B+2},
$$

and so

$$
N \leqslant q^{B(2 n-1)+r+6} \leqslant q^{(n(n-1) / 2-1) / 2} \leqslant|S|^{1 / 2} .
$$

Consider the case $L=S p_{n}(q)$, so $n=2 r$ and $m$ are even. Then $\mathbf{C}_{L}(g)$ contains $S p_{m}(q)$. It follows that

$$
\left|g^{L}\right| \leqslant \frac{\left|S p_{n}(q)\right|}{\left|S p_{m}(q)\right|}<\frac{q^{n(n+1) / 2}}{q^{m(m+1) / 2} / 2}=2 q^{(n-m)(n+m+1) / 2+1} \leqslant q^{(2 n+1) B+2},
$$


and so

$$
N \leqslant q^{B(2 n+1)+r+6} \leqslant q^{(n(n+1) / 2-1) / 2} \leqslant|S|^{1 / 2} .
$$

THEOREM 3.8. Theorem 3.1 holds for all simple classical groups of sufficiently large rank.

Proof. (a) View $S=G / \mathbf{Z}(G)$ with $G=\mathcal{G}^{F}$ as above, and let $r:=\operatorname{rank}(\mathcal{G})$. We will show that there are some $r_{0}=r_{0}\left(w_{1}, w_{2}\right)>8$ and $B=B\left(w_{1}, w_{2}\right)$ such that Theorem 3.1 holds when $r \geqslant r_{0}$, for suitable regular semisimple elements $s_{1}$, $s_{2} \in S$ and with $S_{1}$ being the set of elements in $S$ of support $<B$. By Lemma 3.7, $\left|S_{1}\right| \leqslant|S|^{1 / 2}$ if $r_{0} \geqslant 8 B+3$.

Again, note that, for any regular semisimple element $h \in G, \mathbf{C}_{\mathcal{G}}(h)$ is a maximal torus (as $\mathcal{G}$ is simply connected), and so $\left|\mathbf{C}_{G}(h)\right| \leqslant(q+1)^{r}$. It follows that $\left|\mathbf{C}_{G}(h \mathbf{Z}(G))\right| \leqslant(q+1)^{r}|\mathbf{Z}(G)|$, and so $\left|\mathbf{C}_{S}(h \mathbf{Z}(G))\right| \leqslant(q+1)^{r}$. Also, $|G|>q^{r(r+1)}$ and $|\mathbf{Z}(G)| \leqslant r+1$. So when $r \geqslant r_{0}>8$ we have

$$
\left|\mathbf{C}_{S}(h \mathbf{Z}(G))\right| \leqslant(q+1)^{r}<\left(\frac{q^{r(r+1)}}{r+1}\right)^{1 / 3}<|S|^{1 / 3} .
$$

In particular, $s_{1}$ and $s_{2}$ satisfy condition (iii) of Theorem 3.1 when $r_{0} \geqslant 9$. As mentioned above, condition (i) of Theorem 3.1 follows from [LST, Theorem 1.1.1]. So it suffices to establish (3.1) for all $g \in S \backslash S_{1}$.

(b) Suppose first that $\mathcal{G}^{F}$ is a special linear, special unitary, or symplectic group. By Propositions 6.2.4 and 6.1.1 of [LST], there is some $r_{1}=r_{1}\left(w_{1}, w_{2}\right)$ with the following property. When $r \geqslant r_{1}$, there are regular semisimple elements $s_{i} \in$ $w_{i}(S)$ for $i=1,2$ such that there are at most $\kappa \leqslant 4$ irreducible characters $\chi_{i} \in$ $\operatorname{Irr}(S)$ with $\chi_{i}\left(s_{1}\right) \chi_{i}\left(s_{2}\right) \neq 0,1 \leqslant i \leqslant \kappa$, and $\chi_{1}=1_{S}$. Moreover, $\left|\chi_{i}\left(s_{1}\right) \chi_{i}\left(s_{2}\right)\right|=1$ for $1 \leqslant i \leqslant \kappa$. Now we choose $B \geqslant 1443^{2}$ and consider any $g \in S \backslash S_{1}$. By [LST, Theorem 1.2.1],

$$
\frac{|\chi(g)|}{\chi(1)}<q^{-\sqrt{B} / 481}<q^{-3} \leqslant 1 / 8,
$$

whence

$$
\left|\sum_{1_{S} \neq \chi \in \operatorname{Irr}(S)} \frac{\chi\left(s_{1}\right) \chi\left(s_{2}\right) \bar{\chi}(g)}{\chi(1)}\right| \leqslant \sum_{i=2}^{\kappa} \frac{\left|\chi_{i}(g)\right|}{\chi_{i}(1)}<3 / 8,
$$

as required. In fact, if $\mathcal{G}^{F}$ is a symplectic group, then $\kappa=2, \chi_{2}=\mathrm{St}$, $\left|\chi_{2}(g) / \chi(1)\right| \leqslant 1 / q \leqslant 1 / 2$ for all $1 \neq g \in S$, and so we can take $S_{1}=\{1\}$.

(c) Suppose now that $\mathcal{G}^{F}$ is a simple orthogonal group. By Propositions 6.3.5 and 6.3 .7 of [LST], there exist some $r_{2}=r_{2}\left(w_{1}, w_{2}\right), \kappa=\kappa\left(w_{1}, w_{2}\right)$, and $C=C\left(w_{1}, w_{2}\right)$ with the following property. When $r \geqslant r_{2}$, there are regular 
semisimple elements $s_{i} \in w_{i}(S)$ for $i=1,2$ such that there are at most $\kappa$ irreducible characters $\chi_{i} \in \operatorname{Irr}(S)$ with $\chi_{i}\left(s_{1}\right) \chi_{i}\left(s_{2}\right) \neq 0,1 \leqslant i \leqslant \kappa$, and $\chi_{1}=1_{S}$. Moreover, $\left|\chi_{i}\left(s_{1}\right) \chi_{i}\left(s_{2}\right)\right| \leqslant C$ for $1 \leqslant i \leqslant \kappa$. Now we choose $B \geqslant 1443^{2}$ such that

$$
(\kappa-1) C^{2} 2^{-\sqrt{B} / 481}<1 / 2 .
$$

Then, for any $g \in S \backslash S_{1}$, by [LST, Theorem 1.2.1], we have

$$
\left|\sum_{1_{S} \neq \chi \in \operatorname{Irr}(S)} \frac{\chi\left(s_{1}\right) \chi\left(s_{2}\right) \bar{\chi}(g)}{\chi(1)}\right| \leqslant \sum_{i=2}^{\kappa} \frac{C^{2}\left|\chi_{i}(g)\right|}{\chi_{i}(1)}<(\kappa-1) C^{2} 2^{-\sqrt{B} / 481}<1 / 2 .
$$

Hence we are done by choosing $r_{0}:=\max \left(r_{1}, r_{2}, 9,8 B+3\right)$.

\section{Alternating groups}

Suppose that $G$ is a group and that $X$ and $Y$ are subsets. If we have subsets $X_{1}, \ldots, X_{k} \subseteq X, Y_{i}, \ldots, Y_{k} \subseteq Y$, and $Z_{1}, \ldots, Z_{k} \subseteq Z$ such that $Z_{i} \subseteq X_{i} Y_{i}$ and $\cup Z_{i}=G$, then, setting $X_{0}=X_{1} \cup \cdots \cup X_{k}$ and $Y_{0}=Y_{1} \cup \cdots \cup Y_{k}$, we have $X_{0} Y_{0}=G$. We use this construction to find $X_{0} \subseteq w_{1}\left(\mathrm{~A}_{n}\right)$ and $Y_{0} \subseteq w_{2}\left(\mathrm{~A}_{n}\right)$ such that $X_{0} Y_{0}=\mathrm{A}_{n}$ and $\left|X_{0}\right|,\left|Y_{0}\right|$ are of order $n !^{1 / 2} \sqrt{\log n !}$.

We begin by noting that, for any word $w$ and any group $G, w(G)$ is a characteristic set, that is, invariant under every automorphism of $G$. In particular, $w\left(\mathrm{~A}_{n}\right)$ is a union of $\mathrm{S}_{n}$-conjugacy classes. If $g_{1}, g_{2} \in \mathrm{A}_{n}$ and $C_{1}$ and $C_{2}$ denote their $\mathrm{S}_{n}$-conjugacy classes, then

$$
\left|\left\{\left(c_{1}, c_{2}\right) \in C_{1} \times C_{2} \mid c_{1} c_{2}=g\right\}\right|=\frac{\left|C_{1}\right|\left|C_{2}\right|}{n !} \sum_{\chi} \frac{\chi\left(g_{1}\right) \chi\left(g_{2}\right) \bar{\chi}(g)}{\chi(1)} .
$$

We recall a basic upper bound estimate [LS1, Theorem 1.1] for $|\chi(g)|$. For $g \in \mathrm{S}_{n}$ and $i \in \mathbb{N}$, let $\Sigma_{i}(g)$ denote the union of all $g$-cycles of length $\leqslant i$ in $\{1, \ldots, n\}$. Define $e_{1}(g), e_{2}(g), \ldots$ so that

$$
n^{e_{1}(g)+\cdots+e_{i}(g)}=\max \left(1,\left|\Sigma_{i}(g)\right|\right)
$$

for all $i \in \mathbb{N}$. Define

$$
E(g)=\sum_{i=1}^{\infty} \frac{e_{i}(g)}{i}
$$

Then for all $\epsilon>0$ there exists $N$ such that, for all $n>N$, all $g \in \mathrm{S}_{n}$, and all irreducible characters $\chi$ of $\mathrm{S}_{n}$,

$$
|\chi(g)| \leqslant|\chi(1)|^{E(g)+\epsilon} .
$$


For example, if $g$ has a bounded number of cycles, and $n$ is sufficiently large in terms of $\epsilon$,

$$
|\chi(g)| \leqslant|\chi(1)|^{\epsilon} .
$$

If $g$ has no more than $n^{2 / 3}$ fixed points and $n$ is sufficiently large in terms of $\epsilon$, then

$$
|\chi(g)| \leqslant|\chi(1)|^{5 / 6+\epsilon} .
$$

By a result of Liebeck and Shalev [LiS, Theorem 1.1], for all $s>0$,

$$
\lim _{n \rightarrow \infty} \sum_{\chi \in \operatorname{Irr}\left(\mathrm{S}_{n}\right)} \chi(1)^{-s}=2 .
$$

Note that the trivial character and the sign character each contribute 1 to the above sum; excluding them from the sum, the limit would be zero. Of course, thus if $g_{1}, g_{2}$, and $g$ are all even permutations, then the trivial character and the sign character each contribute $\left(\left|C_{1}\right|\left|C_{2}\right|\right) / n$ ! to expression (4.1). From this, we conclude the following.

Proposition 4.1. For all $\epsilon>0$ and integers $k_{1}$ and $k_{2}$, there exists an integer $N=N\left(\epsilon, k_{1}, k_{2}\right)$ such that, if $n>N$ and $C_{1}$ and $C_{2}$ are even conjugacy classes in $\mathrm{S}_{n}$ consisting of $k_{1}$ and $k_{2}$ cycles, respectively, then every $g \in \mathrm{A}_{n}$ with no more than $n^{2 / 3}$ fixed points is represented in at least

$$
(1-\epsilon) \frac{\left|C_{1}\right|\left|C_{2}\right|}{\left|\mathrm{A}_{n}\right|}
$$

different ways as $x_{1} x_{2}, x_{1} \in C_{1}, x_{2} \in C_{2}$.

Now, by [LS2, Theorem 1.3], if $n$ is sufficiently large, $w_{1}\left(\mathbf{A}_{n}\right)$ and $w_{2}\left(\mathbf{A}_{n}\right)$ each contain elements $g_{1}$ and $g_{2}$, respectively, with at most 6 cycles of length $>1$ and $\leqslant 17$ cycles in total. So there is some constant $A$ such that $\left|\mathbf{C}_{\mathrm{S}_{n}}\left(g_{i}\right)\right|<A n^{6}$ for $i=1,2$, whence

$$
\left|w_{i}\left(\mathbf{A}_{n}\right)\right| \geqslant\left|\left(g_{i}\right)^{S_{n}}\right|>2 e(n !)^{1 / 2} \log ^{1 / 2} n ! .
$$

Defining $Z_{1}$ as the set of elements of $\mathrm{A}_{n}$ with no more than $n^{2 / 3}$ fixed points, it follows from Proposition 2.3 that there exist $X_{1}$ and $Y_{1}$ contained in $w_{1}\left(\mathrm{~A}_{n}\right)$ and $w_{2}\left(\mathrm{~A}_{n}\right)$, respectively, such that $Z_{1} \subseteq X_{1} Y_{1}$.

What remains is to define $X_{i}, Y_{i}, Z_{i}$ for $i \geqslant 2$ to cover the elements of $\mathrm{A}_{n}$ with more than $n^{2 / 3}$ fixed points.

The number of elements of $A_{n}$ with at least $m:=\lceil 2 n / 3\rceil$ fixed points is less than

$$
\sum_{i=m}^{n}\left(\begin{array}{l}
n \\
i
\end{array}\right)(n-i) !=\sum_{i=m}^{n} \frac{n !}{i !}<2 \frac{n !}{m !} \leqslant n !^{1 / 3+o(1)} .
$$


Therefore, we can represent each element $g$ with at least $m$ fixed points as $x_{g} y_{g}$, $x_{g} \in w_{1}\left(\mathrm{~A}_{n}\right), y_{g} \in w_{2}\left(\mathrm{~A}_{n}\right)$, and we can define $X_{2}$ to be the union of all such $x_{g}$ and $Y_{2}$ the union of all such $y_{g}$. Note that

$$
\left|X_{2}\right|,\left|Y_{2}\right|<(n !)^{1 / 3+o(1)} \text {. }
$$

This reduces the problem to elements $g$ with

$$
n^{2 / 3} \leqslant|\operatorname{Fix}(g)| \leqslant 2 n / 3 .
$$

For each $T \subseteq\{1,2, \ldots, n\}$ with $m:=|T| \in\left[n^{2 / 3}, 2 n / 3\right]$, we define $\mathrm{S}_{T} \subseteq \mathrm{S}_{n}$ to be the pointwise stabilizer of $T$ in $\mathrm{S}_{n}$ and $\mathrm{A}_{T}$ to be the pointwise stabilizer of $T$ in $\mathrm{A}_{n}$. Thus $\mathrm{S}_{T}$ is isomorphic to $\mathrm{S}_{n-m}$ and $\mathrm{A}_{T}$ is isomorphic to $\mathrm{A}_{n-m}$, where $n-m \in\left[n / 3, n-n^{2 / 3}\right]$. For each $T$, we choose an $\mathrm{S}_{T}$-conjugacy class $C_{1, T}$ in $w_{1}\left(\mathbf{A}_{T}\right)$ and an $\mathbf{S}_{T}$-conjugacy class $C_{2, T}$ in $w_{2}\left(\mathbf{A}_{T}\right)$, each consisting of at most 17 cycles when regarded as elements of $\mathrm{S}_{n-m}$. (Of course there are $|T|$ additional 1-cycles when we regard them as elements of $\mathrm{S}_{n}$.) If $n$ is sufficiently large, $n-m$ is larger than the constant $N$ of Proposition 4.1, and we conclude that every fixed point free element of $\mathrm{A}_{n-m}$ can be written in at least

$$
(1-\epsilon) \frac{\left|C_{1, T}\right|\left|C_{2, T}\right|}{\left|\mathrm{A}_{n-m}\right|}
$$

ways. Applying Proposition 2.3 and arguing as above, we conclude that there exist subsets $X_{T}$ and $Y_{T}$ of $C_{1, T}$ and $C_{2, T}$, respectively, such that $X_{T} Y_{T}$ contains all elements of $\mathrm{S}_{n}$ with fixed point set exactly $T$, and $\left|X_{T}\right|$ and $\left|Y_{T}\right|$ are bounded above by

$$
c(n-m) !^{1 / 2} \log ^{1 / 2}(n-m) !
$$

where $c$ is independent of $n$ or $m$. An upper bound for the cardinality of $\bigcup_{T} X_{T}$ is

$$
\begin{aligned}
& c n \log n \sum_{n^{2 / 3} \leqslant m \leqslant 2 n / 3}\left(\begin{array}{l}
n \\
m
\end{array}\right)(n-m) !^{1 / 2} \\
& \leqslant c n^{3} \max \left\{\left(\begin{array}{l}
n \\
m
\end{array}\right)(n-m) !^{1 / 2} \mid n^{2 / 3} \leqslant m \leqslant 2 n / 3\right\},
\end{aligned}
$$

and likewise for $\bigcup_{T} Y_{T}$.

For $m \geqslant n^{2 / 3}$, we have by Stirling's approximation

$$
m !>(m / e)^{m} .
$$

So, when $n>\left(2 e^{2}\right)^{3}$ is large enough, we have that

$$
\begin{aligned}
\frac{\left(\begin{array}{c}
n \\
m
\end{array}\right) \cdot(n-m) !^{1 / 2}}{(n !)^{1 / 2}} & =\frac{\left(\prod_{j=n-m+1}^{n} j\right)^{1 / 2}}{m !}<\frac{n^{m / 2}}{e^{-m} m^{m}} \\
& =\left(\frac{e^{2} n}{m^{2}}\right)^{m / 2}<\left(\frac{e^{2}}{n^{1 / 3}}\right)^{\left(n^{2 / 3}\right) / 2}<\left(\frac{1}{2}\right)^{\left(n^{2 / 3}\right) / 2}<\frac{1}{c n^{3}} .
\end{aligned}
$$


In this case, the cardinalities of $\bigcup_{T} X_{T}$ and $\bigcup_{T} Y_{T}$ are less than $n !^{1 / 2}$. It follows that $X_{1}, X_{2}$, and all the $X_{T}$ together have cardinality $O\left((n !)^{1 / 2} \log ^{1 / 2} n\right)$, and likewise for $Y$. That concludes the proof of Theorem 1.1 in the alternating case.

\section{Groups as products of two subsets}

LEMMA 5.1. Let $G$ be a cyclic group of prime order $p$, and $x$ any real number with $2 \leqslant x \leqslant p$. Then there exist subsets $X$ and $Y$ of $G$ with $|X| \leqslant x$ and $|Y| \leqslant$ $2 p / x$ such that $X Y=G$.

Proof. Identify $G$ with the additive group $\mathbb{Z} / p \mathbb{Z}$ and its elements with $0,1, \ldots$, $p-1$. The cases $2 \leqslant p \leqslant 7$ are obvious, so we will assume that $p \geqslant 11$. Since the roles of $x$ and $2 p / x$ are symmetric, we may assume that $x \geqslant \sqrt{2 p}>4$. Now if $x \geqslant p-2$ then $G=X+Y$ with $X:=\{2 j \mid 0 \leqslant j \leqslant(p-1) / 2\}$ and $Y=\{0,1\}$. Suppose that $p-2>x \geqslant \sqrt{2 p}$. Setting $a:=\lfloor x\rfloor \leqslant x$ and $b:=\lceil p / a\rceil \geqslant p / a$, we see that $b<\max (p / a+1,2 p / x)$ and $G=X+Y$ for

$$
X:=\{0,1, \ldots, a-1\}, \quad Y=\{j a \mid 0 \leqslant j \leqslant b-1\} .
$$

LEMMA 5.2. Let $G$ be a finite nonabelian simple group of order $n$. Then $G$ possesses a maximal subgroup $M$, with $|M| \geqslant \sqrt{n}$ if $G=J_{3}$ and $|M| \geqslant \sqrt{2 n}$ otherwise.

Proof. The case of 26 sporadic simple groups can be checked using [Atlas]. If $G=\mathrm{A}_{n}$ with $n \geqslant 5$, take $M:=\mathrm{A}_{n-1}$. So we may assume that $G$ is a finite simple group of Lie type. If $G$ is a classical group, then the smallest index of proper subgroups of $G$ is listed in [KL, Table 5.2.A], whence the statement follows. If $G$ is an exceptional group, then [MMT, Table 3.5] lists a subgroup $N$ of $G$, and one can check that $|N| \geqslant \sqrt{2 n}$.

THEOREM 5.3. Let $G$ be any finite group of order $n$, and $x$ any real number with $2 \leqslant x \leqslant n$. Then there exist subsets $X$ and $Y$ of $G$ with $|X| \leqslant x$ and $|Y| \leqslant 2 n / x$ such that $X Y=G$.

Proof. We proceed by induction on $|G|$. Note that the roles of $x$ and $y:=2 n / x$ in the statement are symmetric, and so without loss of generality we may assume that $x \leqslant y$, that is $x \leqslant \sqrt{n / 2}$.

(a) Suppose that there is a subgroup $H<G$ with $|H|>x$. By the induction hypothesis, there exist subsets $X^{\prime}, Y^{\prime} \subseteq H$ with $X^{\prime} Y^{\prime}=H,\left|X^{\prime}\right| \leqslant x$, and $\left|Y^{\prime}\right| \leqslant$ $2|H| / x$. Decompose $G=\bigcup_{i=1}^{m} H y_{i}$ with $m=[G: H]$, and let $X:=X^{\prime}$ and $Y:=\bigcup_{i=1}^{m} Y^{\prime} y_{i}$. Then $X Y=G,|X| \leqslant x$, and $|Y| \leqslant m\left|Y^{\prime}\right| \leqslant 2|G| / x$. 
Next, let us consider the possibility that $H<G$ is a subgroup with $x / 2 \leqslant$ $|H|<x$. Then setting $X:=H$ and $Y$ a set of coset representatives of $H$ in $G$, we get $G=X Y,|X| \leqslant x$, and $|Y|=[G: H] \leqslant 2 n / x$.

Thus we are done if $G$ possesses a proper subgroup of order $\geqslant x / 2$.

(b) Suppose now that $G$ admits a nontrivial normal subgroup $H$ with $|H|<$ $x / 2$. By the induction hypothesis applied to $G / H$ and $x^{\prime}:=x /|H|$, there exist subsets $X^{\prime}, Y^{\prime} \subseteq G / H$ with $\left|X^{\prime}\right| \leqslant x^{\prime},\left|Y^{\prime}\right| \leqslant 2|G / H| / x^{\prime}=2 n / x$, and $X^{\prime} Y^{\prime}=$ $G / H$. Now let $X$ denote the full inverse image of $X^{\prime}$ in $G$, and let $Y$ denote a set of coset representatives in $G$ for $Y^{\prime}$. Then $G=X Y,|X|=\left|X^{\prime}\right| \cdot|H| \leqslant x$, and $|Y|=\left|Y^{\prime}\right| \leqslant 2 n / x$.

(c) Assume that $G$ is not simple: $1 \neq N \triangleleft G$ for some $N<G$. If $|N| \geqslant x / 2$, then we are done by (a). Otherwise, we are done by (b).

It remains to consider the case when $G$ is simple. If $G$ is abelian, then we can apply Lemma 5.1. Otherwise, by Lemma 5.2 there is a maximal subgroup $M<G$ of order $\geqslant \sqrt{n}>x / 2$, and so we are again done by (a).

COROLlary 5.4. Any finite group $G$ admits a square root $R$, that is, a subset $R \subseteq G$ such that $R^{2}=G$, with $|R| \leqslant \sqrt{8|G|}$.

Proof. Taking $x=\sqrt{2|G|}$ in Theorem 5.3, we see that $G=X Y$ with $|X|$, $|Y| \leqslant x$. Now set $R:=X \cup Y$.

\section{Square roots of a Lie group}

In this section we show that the results of Section 5 extend in a suitable sense to compact Lie groups. We would like to say that the minimum dimension of a square root of $G$ is half the dimension of $G$, but we need a suitable definition of dimension. Hausdorff dimension does not do the job; indeed, it is not difficult to see that $S^{1}$ can be written as $X Y$, where $X$ and $Y$ are both of Hausdorff dimension 0 . It turns out that upper Minkowski dimension is the better notion for our purposes.

We begin by recalling some basic definitions. A good reference is [Ta]. For $\delta>0$, we define the $\delta$-packing number of a bounded metric space $X, N_{\delta}(X)$, to be the maximum number of disjoint open balls of radius $\delta$ in $X$. We recall that the upper Minkowski dimension, $\overline{\operatorname{dim}} X$, of a bounded metric space $X$ is given by the formula

$$
\overline{\operatorname{dim}} X=\limsup _{\delta>0} \frac{-\log N_{\delta}(X)}{\log \delta} .
$$

If $\phi: X \rightarrow Y$ is a surjective Lipschitz map with constant $L$, then $N_{L \delta}(Y) \leqslant$ $N_{\delta}(X)$, so $\overline{\operatorname{dim}} \phi(X) \leqslant \overline{\operatorname{dim}} X$. 
If $[-1,1]$ is endowed with the usual metric $d(x, y)=|x-y|$, then

$$
N_{\delta}([-1,1])=\lfloor 1 / \delta\rfloor,
$$

and it follows that $\overline{\operatorname{dim}}[-1,1]=1$. If the ring $\mathbb{Z}_{p}$ of $p$-adic integers is endowed with the usual metric $d(x, y)=|x-y|_{p}$, it follows that

$$
N_{\delta}\left(\mathbb{Z}_{p}\right)=p^{\max \left(0,1+\left\lfloor-\log _{p} \delta\right\rfloor\right)},
$$

so $\overline{\operatorname{dim}} \mathbb{Z}_{p}=1$.

Upper Minkowski dimension is well suited to our purposes because of the following elementary proposition, which is well known for subsets of Euclidean spaces [Ma, 8.10-8.11].

Proposition 6.1. Let $\left(X, d_{X}\right)$ and $\left(Y, d_{Y}\right)$ be bounded metric spaces, and let $d$ be a metric on $X \times Y$ such that

$$
\max \left(d_{X}\left(x_{1}, x_{2}\right), d_{Y}\left(y_{1}, y_{2}\right)\right) \leqslant d\left(\left(x_{1}, y_{1}\right),\left(x_{2}, y_{2}\right)\right) \leqslant d_{X}\left(x_{1}, x_{2}\right)+d_{Y}\left(y_{1}, y_{2}\right) .
$$

Then

$$
\overline{\operatorname{dim}} X \times Y \leqslant \overline{\operatorname{dim}} X+\overline{\operatorname{dim}} Y,
$$

with equality if $\log N_{\delta}(X) / \log \delta$ and $\log N_{\delta}(Y) / \log \delta$ both converge as $\delta \rightarrow 0$.

Proof. If $x_{1}, \ldots, x_{m}$ are the centers of a maximal collection of disjoint open balls of radius $\delta$ in $X$, then balls of radius $2 \delta$ centered at $x_{1}, \ldots, x_{m}$ cover $X$, and likewise for $Y$. The product of any ball of radius $2 \delta$ in $X$ and any ball of radius $2 \delta$ in $Y$ is contained in some ball of radius $4 \delta$ in $X \times Y$, so $X \times Y$ can be covered by $N_{\delta}(X) N_{\delta}(Y)$ balls of radius $4 \delta$. Given any disjoint collection of balls of radius $4 \delta$ in $X \times Y$, no two centers can lie in the same ball of radius $4 \delta$. Thus,

$$
N_{4 \delta}(X \times Y) \leqslant N_{\delta}(X) N_{\delta}(Y),
$$

which proves (6.1). On the other hand, if $x_{1}, \ldots, x_{m}$ are centers of disjoint balls of radius $\delta$ in $X$ and $y_{1}, \ldots, y_{n}$ are centers of disjoint balls of radius $\delta$ in $Y$, then $\left(x_{i}, y_{j}\right)$ are the centers of disjoint balls of radius $\delta$ in $X \times Y$, so

$$
N_{\delta}(X \times Y) \geqslant N_{\delta}(X) N_{\delta}(Y) .
$$

It follows that

$$
\lim _{\delta \rightarrow 0} \frac{-\log N_{\delta}(X \times Y)}{\log \delta}=\lim _{\delta \rightarrow 0} \frac{-\log N_{\delta}(X)}{\log \delta}+\lim _{\delta \rightarrow 0} \frac{-\log N_{\delta}(Y)}{\log \delta}
$$

if both limits on the right-hand side exist. 
Now let $G$ be a compact Lie group. We say that a metric $d$ on $G$ is compatible if it is left invariant and right invariant by $G$ and there exists a coordinate map from some open neighborhood of the identity $e$ of $G$ to some open set in $\mathbb{R}^{n}$ which is Lipschitz in some neighborhood of $e$. If this is true for some coordinate map, it is true for all coordinate maps at $e$, since smooth maps between open sets in $\mathbb{R}^{n}$ are locally Lipschitz. Likewise, a compatible metric on a compact $p$-adic Lie group is a translation-invariant metric for which there exists a coordinate map from some open neighborhood of $e$ to some open set in $\mathbb{Q}_{p}^{n}$, and the choice of coordinate map does not matter. We recall [Bo, III, Section 4, no. 3] that every real (respectively, $p$-adic) Lie group admits an exponential map from a neighborhood of 0 in $\mathbb{R}^{n}$ (respectively, $\mathbb{Q}_{p}^{n}$ ) which is bijective and whose inverse is a coordinate map.

Proposition 6.2. Let $G$ be a compact Lie group endowed with a compatible metric. Then $\overline{\operatorname{dim}} G$ coincides with the usual topological dimension of $G$.

Proof. By Proposition 6.1, $\overline{\operatorname{dim}} I^{n}=n$, where $I$ is any open interval in $\mathbb{R}$, and it follows that $\overline{\operatorname{dim}} U=n$ for any bounded open set in $\mathbb{R}^{n}$. If $\phi: U \rightarrow G$ is a biLipschitz coordinate map, then $U^{\prime}:=\phi(U)$ is an open subset of $G$ of dimension $n$. Therefore, any translate of $U^{\prime}$ in $G$ has dimension $n$, and likewise for any finite union of such translates. By compactness, $G$ itself is such a union, so $\overline{\operatorname{dim}} G=$ $\operatorname{dim} G$.

There is also a $p$-adic version of the same proposition, whose proof is the same.

Proposition 6.3. Let $G$ be a compact p-adic Lie group endowed with a compatible metric. Then $\overline{\operatorname{dim}} G$ coincides with the usual topological dimension of $G$.

We can now prove our lower bound for square roots of a real or $p$-adic Lie group.

PROPOSITION 6.4. If $X$ and $Y$ are subsets of a compact real or p-adic Lie group $G$ endowed with a compatible metric $d$ and $X Y=G$, then $\overline{\operatorname{dim}} X+\overline{\operatorname{dim}} Y \geqslant$ $\operatorname{dim} G$. In particular, if $X$ is a square root of $G, \overline{\operatorname{dim}} X \geqslant(\operatorname{dim} G) / 2$.

Proof. Defining the metric $e$ on $G \times G$ by

$$
e\left(\left(g_{1}, h_{1}\right),\left(g_{2}, h_{2}\right)\right):=d\left(g_{1}, g_{2}\right)+d\left(h_{1}, h_{2}\right),
$$

we have

$$
d\left(g_{1} h_{1}, g_{2} h_{2}\right) \leqslant d\left(g_{1} h_{1}, g_{1} h_{2}\right)+d\left(g_{1} h_{2}, g_{2} h_{2}\right)=e\left(\left(g_{1}, h_{1}\right),\left(g_{2}, h_{2}\right)\right) .
$$


Thus, the multiplication map $m: G \times G \rightarrow G$ is Lipschitz. It follows that

$$
\overline{\operatorname{dim}} X Y=\overline{\operatorname{dim}} m(X \times Y) \leqslant \overline{\operatorname{dim}} X \times Y \leqslant \overline{\operatorname{dim}} X+\overline{\operatorname{dim}} Y .
$$

If $X Y=G$, then

$$
\overline{\operatorname{dim}} X+\overline{\operatorname{dim}} Y \geqslant \overline{\operatorname{dim}} G=\operatorname{dim} G .
$$

The more interesting direction is the converse.

THEOREM 6.5. Let $G$ be a compact real or p-adic Lie group, endowed with a compatible metric. Then $G$ has a square root of dimension $(\operatorname{dim} G) / 2$.

Proof. Let $G$ be a real (respectively, $p$-adic) Lie group, $L$ the Lie algebra, and exp the exponential map from a neighborhood $U$ of 0 in $L$ to a neighborhood $N$ of $e \in G$. Let $v \in L$ be a sufficiently small nonzero element, specifically, an element satisfying $[-1,1] v \subset U$ (respectively, $\mathbb{Z}_{p} v \subset U$ ). Then the function $e_{v}:[-1$, $1] \rightarrow G$ (respectively, $e_{v}: \mathbb{Z}_{p} \rightarrow G$ ) defined by $e_{v}(t)=\exp (t v)$ is Lipschitz. Let $C_{v}$ denote the image of $e_{v}$.

Choose a basis $v_{1}, \ldots, v_{n}$ of sufficiently small vectors in $L$. If $n=2 k$, let $X_{0}=C_{v_{1}} \cdots C_{v_{k}}$ and $Y=C_{v_{k+1}} \cdots C_{v_{2 k}}$. As $X_{0}$ and $Y$ are each images of sets of dimension $k$ under Lipschitz maps, $\overline{\operatorname{dim}} X_{0}, \overline{\operatorname{dim}} Y \leqslant k=(\operatorname{dim} G) / 2$. On the other hand, $X_{0} Y$ contains a neighborhood of $e$ in $G$, so, letting $X$ denote a suitable finite union of left translates of $X$, we have $X Y=G$ and $\overline{\operatorname{dim}} X \leqslant k$. Thus $X \cup Y$ is a square root of $G$ of dimension $(\operatorname{dim} G) / 2$.

If $n=2 k+1$, we observe that there exist subsets $A$ and $B$ of $[-1,1]$ such that $\overline{\operatorname{dim}} A=\overline{\operatorname{dim}} B=1 / 2$ and $A+B=[-1,1]$. We can take, for instance, the Cantor sets

$$
A=-a_{0}+\sum_{i=1}^{\infty} a_{i} 4^{-i}, \quad a_{i} \in\{0,1\} ; \quad B=\sum_{i=1}^{\infty} b_{i} 4^{-i}, \quad b_{i} \in\{0,2\} .
$$

Likewise, there exist $A, B \subset \mathbb{Z}_{p}$ of dimension $1 / 2$ such that $A+B=\mathbb{Z}_{p}$, for instance,

$$
\begin{gathered}
A=\sum_{i=1}^{\infty} a_{i} p^{2 i}, \quad a_{i} \in\{0,1, \ldots, p-1\} \\
B=\sum_{i=1}^{\infty} b_{i} p^{2 i}, \quad b_{i} \in\{0, p, 2 p, \ldots,(p-1) p\} .
\end{gathered}
$$

Now, setting

$$
X_{0}=C_{v_{1}} \cdots C_{v_{k}} \exp \left(A v_{k+1}\right), \quad Y=\exp \left(B v_{k+1}\right) C_{v_{k+2}} \cdots C_{v_{2 k+1}},
$$


we see that

$$
X_{0} Y=C_{v_{1}} \cdots C_{v_{2 k+1}}
$$

contains a neighborhood of $e$, while $\overline{\operatorname{dim}} X_{0}, \overline{\operatorname{dim}} Y \leqslant k+1 / 2$. The rest of the argument goes as before.

\section{Acknowledgements}

Michael Larsen was partially supported by NSF grant DMS-1101424 and a Simons Fellowship. Pham Huu Tiep was partially supported by NSF grant DMS1201374 and the Simons Foundation Fellowship 305247.

\section{References}

[Bo] N. Bourbaki, 'Éléments de Mathématique. Fasc. XXXVII', in: Groupes et Algèbres de Lie. Chapitre II: Algèbres de Lie Libres. Chapitre III: Groupes de Lie, Actualités Scientifiques et Industrielles, No. 1349 (Hermann, Paris, 1972).

$[\mathrm{Bu}]$ R. Burkhardt, 'Über die Zerlegungszahlen der Suzukigruppen $\operatorname{Sz}(q)$ ', J. Algebra 59 (1979), 421-433.

[Atlas] J. H. Conway, R. T. Curtis, S. P. Norton, R. A. Parker and R. A. Wilson, An ATLAS of Finite Groups (Clarendon, Oxford, 1985).

[DL] P. Deligne and G. Lusztig, 'Representations of reductive groups over finite fields', Ann. of Math. (2) 103(1) (1976), 103-161.

[EG] E. W. Ellers and N. Gordeev, 'On the conjectures of J. Thompson and O. Ore', Trans. Amer. Math. Soc. 350 (1998), 3657-3671.

[FG] J. Fulman and R. M. Guralnick, 'Bounds on the number and sizes of conjugacy classes in finite Chevalley groups with applications to derangements', Trans. Amer. Math. Soc. 364 (2012), 3023-3070.

[GLL] S. Garion, M. Larsen and A. Lubotzky, 'Beauville surfaces and finite simple groups', J. Reine Angew. Math. 666 (2012), 225-243.

[Chevie] M. Geck, G. Hiss, F. Lübeck, G. Malle and G. Pfeiffer, 'CHEVIE-A system for computing and processing generic character tables for finite groups of Lie type, Weyl groups and Hecke algebras', Appl. Algebra Engrg. Comm. Comput. 7 (1996), 175-210.

[GL] R. M. Guralnick and F. Lübeck, 'On $p$-singular elements in Chevalley groups in characteristic p', in: Groups and Computation, III, Vol. 8 (Columbus, OH, 1999) (Ohio State University Mathematical Research Institute Publication, de Gruyter, Berlin, 2001), 169-182.

[GT] R. M. Guralnick and P. H. Tiep, 'Effective results on the Waring problem for finite simple groups', submitted.

[KL] P. B. Kleidman and M. W. Liebeck, The Subgroup Structure of the Finite Classical Groups, London Mathematical Society Lecture Note Series. no. 129 (Cambridge University Press, 1990).

[La] M. Larsen, 'Word maps have large image', Israel J. Math. 139 (2004), 149-156.

[LS1] M. Larsen and A. Shalev, 'Characters of symmetric groups: sharp bounds and applications', Invent. Math. 174(3) (2008), 645-687. 
[LS2] M. Larsen and A. Shalev, 'Word maps and Waring type problems', J. Amer. Math. Soc. 22(2) (2009), 437-466.

[LST] M. Larsen, A. Shalev and P. H. Tiep, 'Waring problem for finite simple groups', Ann. of Math. (2) 174(3) (2011), 1885-1950.

[LBST] M. W. Liebeck, E. O'Brien, A. Shalev and P. H. Tiep, 'The ore conjecture', J. Eur. Math. Soc. 12 (2010), 939-1008.

[LiS] M. W. Liebeck and A. Shalev, 'Fuchsian groups, coverings of Riemann surfaces, subgroup growth, random quotients and random walks', J. Algebra 276 (2004), 552-601.

[Lu1] F. Lübeck, 'Finding $p$ '-elements in finite groups of Lie type', in: Groups and Computation, III, Vol. 8 (Columbus, OH, 1999) (Ohio State University Mathematical Research Institute Publication, de Gruyter, Berlin, 2001), 249-255.

[Lu2] F. Lübeck, Character degrees and their multiplicities for some groups of Lie type of rank $<$ 9, http://www.math.rwth-aachen.de/ Frank.Luebeck/chev/DegMult/index.html.

[LM] F. Lübeck and G. Malle, '(2, 3)-generation of exceptional groups', J. Lond. Math. Soc. 59 (1999), 109-122.

[MMT] K. Magaard, G. Malle and P. H. Tiep, 'Irreducibility of tensor squares, symmetric squares, and alternating squares', Pacific J. Math. 202 (2002), 379-427.

[MSW] G. Malle, J. Saxl and T. Weigel, 'Generation of classical groups', Geom. Dedicata 49 (1994), 85-116.

[Ma] P. Mattila, Geometry of Sets and Measures in Euclidean Spaces. Fractals and Rectifiability, Cambridge Studies in Advanced Mathematics, 44 (Cambridge University Press, Cambridge, 1995).

[Ta] T. Tao, 245C, Notes 5: Hausdorff dimension, http://terrytao.wordpress.com/2009/05/19/ 245c-notes-5-hausdorff-dimension-optional/.

[Vu] H. Van Vu, 'On a refinement of Waring's problem', Duke Math. J. 105(1) (2000), $107-134$.

[Wa] H. N. Ward, 'On Ree's series of simple groups', Trans. Amer. Math. Soc. 121 (1966), 62-89. 